\title{
Checklist das Cryptophyceae do estado de São Paulo, Brasil
}

\author{
Andréa Tucci ${ }^{1,5}$, Carlos Eduardo de Mattos Bicudo ${ }^{2}$, \\ Mariângela Menezes ${ }^{3}$, João Alexandre Saviolo Osti ${ }^{4}$ \& Gisele Adame ${ }^{I}$ \\ ${ }^{1}$ Núcleo de Pesquisa em Ficologia, Instituto de Botânica, \\ Av. Miguel Stefano 3687, CEP 04301-902, São Paulo, SP, Brasil \\ ${ }^{2}$ Núcleo de Pesquisa em Ecologia, Instituto de Botânica, \\ Av. Miguel Stefano 3687, CEP 04301-902, São Paulo, SP, Brasil \\ ${ }^{3}$ Laboratório de Ficologia, Museu Nacional, Universidade Federal do Rio de Janeiro, \\ Quinta da Boa Vista, São Cristovão, CEP 20.940-040, Rio de Janeiro, RJ, Brasil \\ ${ }^{4}$ Centro de Aquicultura, Universidade Estadual Paulista “Julio de Mesquita Filho", \\ Via de Acesso Prof Paulo Donato Castellane, S/N, CEP 14884-900, Jaboticabal, SP, Brasil \\ ${ }^{5}$ Autor para correspondência: Andréa Tucci,e-mail: atuccic@ig.com.br
}

TUCCI, A., BICUDO, C.E.M., MENEZES, M., OSTI, J.A.S. \& ADAME, G. Checklist of Cryptophyceae from São Paulo State, Brazil. Biota Neotrop. 11(1a): http://www.biotaneotropica.org.br/v11n1a/en/abstract?invent ory+bn0101101a2011

\begin{abstract}
Based on the information provided by the monograph on the group published by the project Phycology Flora of São Paulo (BIOTA), the collection deposited in the Scientific Herbarium "Mary P. Eneyda Kauffmann Fidalgo " of the State of São Paulo, the List of Endangered Species of the Flora of Brazil and scientific papers, dissertations and theses (with descriptions and illustrations), there are nine genera and 39 species of cryptophytes, 16 of which are unique species to the State of São Paulo, all recorded in freshwater environments. The lack of specialists in the State of São Paulo and Brazil as well as problems in the sampling strategy, the necessary use of electronic microscopy and the absence of molecular biology studies are factors that must have underestimated the taxonomic knowledge of Cryptophyceae in the state.
\end{abstract}

Keywords: Cryptophyceae, Cryptomonas, biodiversity, BIOTA/FAPESP Program.

Number of species: In the world: 120-150, in Brazil: 40, estimated in São Paulo State: 50

TUCCI, A., BICUDO, C.E.M., MENEZES, M., OSTI, J.A.S. \& ADAME, G. Checklist das Cryptophyceae do Estado de São Paulo, Brasil. Biota Neotrop. 11(1a): http://www.biotaneotropica.org.br/v11n1a/pt/abstract ?inventory+bn0101101a2011

Resumo: Usando como base de informações a monografia sobre o grupo publicada pelo projeto Flora Ficológica do Estado de São Paulo (Programa BIOTA), o acervo depositado no Herbário Científico do Estado de São Paulo "Maria Eneyda P. Kauffmann Fidalgo" (SP), a Lista de Espécies da Flora do Brasil e artigos científicos, dissertações e teses (com descrições e ilustrações) temos: nove gêneros e 39 espécies de criptoficeas, sendo 16 espécies exclusivas para o Estado de São Paulo, todas registradas em ambientes de água doce. A carência de especialistas no Estado de São Paulo e no Brasil, além de problemas de estratégia amostral, necessidade de uso da microscopia eletrônica e ausência de estudos de biologia molecular, são fatores que devem ter subestimado o conhecimento taxonômico de Cryptophyceae no Estado.

Palavras-chave: Cryptophyceae, Cryptomonas, biodiversidade, Programa BIOTA/FAPESP.

Número de espécies: No mundo: 120-150, no Brasil: 40, estimadas no Estado de São Paulo: 50 


\section{Introdução}

As Cryptophyceae constituem uma classe bem definida de algas que engloba organismos unicelulares biflagelados, mixotróficos e ativamente móveis na fase vegetativa, além de alguns poucos cocóides e de hábito dominantemente isolado como em Tetragonidium Pascher e Bjornbergiella C. Bicudo (Castro \& Bicudo 2007).

Representantes dessa classe são facilmente reconhecidas pela forma assimétrica da célula, com nítida dorsiventralidade, ápice deslocado para o lado esquerdo e inserção dos flagelos deslocada para o lado direito. A face dorsal é convexa enquanto que a ventral é plana ou levemente côncava, além de um sulco longitudinal raso. $\mathrm{Na}$ face ventral e, mais especificamente, no pólo anterior da célula, próximo do sulco longitudinal, ocorre uma invaginação ou citofaringe revestida internamente por uma quantidade variável de ejectissômios de disposição linear (Castro \& Bicudo 2007).

O envoltório celular é caracterizado por um periplasto rígido, de natureza protéica, que cobre externamente (componente externo do periplasto) e internamente (componente interno do periplasto) o plasmalema, sendo formado, em geral, por plaquetas retangulares ou poligonais. Os componentes externo e interno do periplasto podem ou não apresentar a mesma morfologia das plaquetas. Algumas vezes, as plaquetas estão ausentes ou presentes apenas em um dos componentes do periplasto. Escamas em roseta e material fibrilar podem ocorrer em adição às plaquetas externas, algumas vezes em substituição a elas (Hoef-Emden \& Melkonian 2003).

Os ejectissômios são organelas "explosivas" situadas imediatamente abaixo do plasmalema, sendo mais desenvolvidos e facilmente observáveis na região da citofaringe e os menores distribuídos ao longo da periferia celular (Hoef-Emden \& Melkonian 2003). Um vacúolo contrátil ocorre no pólo anterior da célula em representantes de água doce. Imediatamente adjacentes à citofaringe ocorrem, às vezes, um ou dois corpos de Maupas, que nada mais são do que estruturas de função ainda desconhecida, embora possivelmente envolvidas na remoção e digestão de membranas supérfluas (Castro \& Bicudo 2007).

Os dois flagelos são desiguais em tamanho e estão inseridos no vestíbulo da citofaringe, deslocados para o lado direito da célula, emergindo subapicalmente acima e à direita da célula e à esquerda da citofaringe (quando se observa a célula em vista ventral). Essa inserção assimétrica dos flagelos confere uma rotação desequilibrada em torno do eixo longitudinal da célula durante o nado, com deslocamento típicamente ondulatório (Hoef-Emden 2007).

Nos representantes pigmentados ocorre um cloroplastídio inteiro ou bilobado e, às vezes, dois parietais. Em Cyanomonas e Pseudocryptomonas ocorrem vários cloroplastídios discóides parietais. Os cloroplastídios são oriundos de uma endosimbiose secundária com uma alga vermelha. Possuem quatro membranas além do núcleo vestigial da alga vermelha ou nucleomorfo localizado no espaço periplastidial, entre as duas membrasnas externas e as duas internas do cloroplastídio. Além das clorofilas $a$ e $c$, os cloroplastídios apresentam as biliproteinas ficoeritrina e ficocianina, $\alpha$-caroteno e carotenóides (aloxantina) como os principais pigmentos acessórios, o que lhes confere coloração variada desde azul a azul-esverdeada, avermelhada, castanho-avermelhada, verde-oliva, castanha até castanho-amarelada. As biliproteinas encontram-se preenchendo o lúmen dos tilacóides, estes últimos organizados no interior dos cloroplastídios, frequentemente aos pares, formando lamelas (Castro \& Bicudo 2007).

Pirenóides podem ocorrer isolados, usualmente no centro do cloroplastídio, na face dorsal da célula, ou aos pares, situados nos lobos dos cloroplastídios. $\mathrm{O}$ amido constitui a principal substância de reserva acumulada no espaço periplastidial. Além de amido, existem gotículas de gordura no citoplasma (Castro \& Bicudo 2007).

O nucleomorfo apresenta posição variada no espaço periplastidial como, por exemplo, em uma invaginação do pirenóide, anteriormente ao núcleo, em uma depressão no núcleo ou entre o núcleo e o pirenóide. Contando apenas com três cromossomos, o nucleomorfo representa uma importante organela na elucidação do processo de redução e compactação do genoma e na filogenia e evolução das Cryptophyceae (Archibald 2007).

Sabe-se, atualmente, que alguns gêneros da classe apresentam reprodução sexuada com histórico de vida dimórfico, com dois diferentes morfotipos. Exemplo: Cryptomonas, que alterna um morfotipo campilomorfo, inicialmente considerado como Campylomonas e um criptomorfo. Dados moleculares têm demonstrado que muitos gêneros estreitamente relacionados com distintas morfologias são, de fato, estádios haplóide e diplóide da mesma espécie e que, aparentemente, um ciclo de vida haplo-diplonte é uma aspecto do grupo como um todo (Hoef-Emden \& Melkonian 2003, Hoef-Emden 2007).

Representantes de criptofíceas são amplamente distribuídos em ambientes tanto marinhos quanto de água doce, que vão desde os pólos até os trópicos e neles representados por aproximadamente o mesmo número de espécies (cerca de 100 em cada um). Nos hábitats pelágicos marinhos, as Cryptophyceae desempenham papel importante na produção primária (formas fotossintéticas) e podem, muitas vezes, formar extensas florações. Nos ambientes de água doce estas algas ocorrem, preferencialmente, em lagos oligotróficos, mas também ocorrem em pequenos corpos d'água e rios com altas concentrações de nutrientes, próximo a macrófitas submersas. Estão presentes praticamente ao longo de todo ano em baixas densidades, ainda que também possam formar florações (Menezes \& Novarino 2003, Novarino 2003).

Embora as Cryptophyceae constituam uma classe de algas bem definida, o sistema de classificação do grupo ainda se encontra indefinido. A delimitação dos gêneros tem sido fundamentada no tipo de ficobiliproteina, na ultrastrutura do periplasto, no tipo de citofaringe e sulco, na localização do nucleomorfo, sendo estes três últimos caracteres definidos apenas por microscopia eletrônica (Novarino 2003). As espécies têm sido definidas, praticamente, com base em caracteres morfológicos visíveis sob microscopia ótica, tais como forma e dimensões celulares, número, localização e forma do cloroplastídio e presença, número e localização de pirenóides. A taxonomia do grupo está baseada, portanto, no conceito clássico de morfoespécie. A descoberta de reprodução sexuada e de um histórico de vida dimórfico em alguns gêneros indica a ocorrência de espécie biológica em Cryptophyceae (Hoef-Emden 2007). Entretanto, as árvores filogenéticas resultantes do sequenciamento genético de cepas de algumas espécies de Cryptomonas mostraram incongruências entre as morfoespécies e as espécies biológicas dentro dos táxons estudados. Cepas geneticamente idênticas ou muito semelhantes entre si mostraram formas celulares que correspondiam a diferentes espécies morfológicas, enquanto que cepas pertencentes a uma mesma morfoespécie foram geneticamente muito diversas, a ponto de representarem espécies biológicas (Hoef-Emden 2007). Os estudos realizados até hoje demonstram que algumas espécies têm seus caracteres morfológicos sustentados por sequenciamento genético, enquanto que outras só podem ser delimitadas por assinaturas moleculares.

O conhecimento das criptofíceas do Estado de São Paulo ainda é bastante incipiente e encontra-se distribuído em 28 publicações com enfoque diversificado, envolvendo estudos taxonômicos, ecológicos ou de hidrobiologia sanitária (Castro \& Bicudo 2007). 
O trabalho de Palmer (1960) constitui a primeira citação de ocorrência de representantes da classe no Estado de São Paulo, no qual o gênero Cryptomonas é apenas listado em meio a outros gêneros de algas de várias outras classes taxonômicas.

Com base em material coletado no hidrofitotério do Jardim Botânico de São Paulo, Bicudo \& Bicudo (1967) só mencionam a presença de duas espécies de criptoficeas, Cryptomonas erosa Ehrenberg e Cryptomonas ovata Ehrenberg, em uma relação de 23 outras de algas pertencentes a seis classes, sem descrevê-las nem ilustrá-las.

Os trabalhos de cunho taxonômico com descrições, ilustrações e, pelo menos, algum comentário dos materiais identificados, estão a seguir. A partir de material oriundo do PEFI, Parque Estadual das Fontes do Ipiranga, município e estado de São Paulo, Bicudo \& Tell (1988) propuseram o gênero Pseudocryptomonas com duas espécies: $P$. americana e $P$. subcylindrica.

O trabalho de Castro et al. (1991) representa uma importante contribuição por ser o primeiro inventário florístico específico de representantes de Cryptophyceae feito para o Estado de São Paulo e, mais especificamente, para a Reserva Biológica do Parque Estadual das Fontes do Ipiranga. Vinte e uma espécies de Chilomonas, Cryptomonas, Pseudocryptomonas, Rhodomonas e Cyathomonas foram identificadas. Constam nesse trabalho descrições e ilustrações, além da proposição de duas espécies novas de Protocryptomonas e uma de Cryptomonas. Cryptomonas foi o gênero que apresentou o maior número de espécies, 10 , sendo $C$. erosa a que apresentou a maior distribuição geográfica no Parque, ocorrendo em seis locais distintos.

Castro \& Bicudo (2007) constitui outro importante e o mais recente trabalho taxonômico, com ilustrações, descrições e comentários a partir de coletas realizadas em vários municípios do Estado. O trabalho é a publicação da tese de doutorado da primeira autora: Castro (1993).

Várias dissertações de mestrado, teses de doutorado e artigos incluem referência à ocorrência de espécies de Cryptophyceae no Estado de São Paulo, a maioria, contudo, de cunho ecológico. São 26 trabalhos, 17 dos quais elaborados a partir de material proveniente de três ambientes situados no PEFI, quais sejam: os lagos das Garças, das Ninféias e do IAG. A grande maioria dos últimos (Ramírez 1996, Nascimento-Moura 1996, Moura 1997, Sant'Anna et al. 1997, Lopes 1999, Vercellino 2001, Crossetti 2002, 2006, Barcelos 2003, Ferragut 2004, Fonseca 2005, Biesemeyer 2005, Fermino 2006) contém unicamente a referência à presença das espécies durante o estudo realizado. Há outros nessa mesma situação, que foram feitos com materiais da Represa de Guapiranga (Beyruth 1996), da Represa Billings (Carvalho 2003, Nishimura 2008), da Represa de Jurumirim (Nogueira 1996, Ferreira 2005, Henry et al. 2006, Granado et al. 2009), da Represa de Barra Bonita (Calijuri 1999) do Reservatório de Salto Grande em Americana (Tucci et al. 2004) e de 30 pesqueiros localizados na região metropolitana da cidade de São Paulo (Gentil 2007). Lopes (1999) resultou em uma publicação (Lopes et al. 2005) cujo conteúdo é o mesmo da parte competente da tese de doutorado; e Crossetti (2002) em duas publicações (Crossetti \& Bicudo 2005a, 2005b) cujos conteúdos também são exatamente os mesmos da dissertação de mestrado.

Cabe ainda mencionar o trabalho de cunho ecológico específico para Cryptophyceae realizado por Bicudo et al. (2009), a partir de material do Lago das Ninféias, no PEFI. Os referidos autores registraram a presença de 10 espécies, das quais, quatro (C. brasiliensis, C. curvata, C. erosa e C. marssonii) apresentaram as maiores densidades populacionais.

Algumas poucas dissertações, teses e artigos apresentaram descrições sucintas (inclusive medidas) e/ou ilustrações dos materiais identificados, permitindo, assim, reidentificação dos materiais nelas incluídos. Neste sentido, Marinho (1994) apresentou breve descrição de Cryptomonas parapyrenoidifera Skuja, C. platyuris Skuja e C. pyrenoidifera Skuja coletadas do Açude do Jacaré, SP. Calijuri (1999) forneceu ilustrações de Cryptomonas pseudopyrenoidifera Skuja e C. tetrapyrenoidosa Skuja, mas, não é possível reidentificar a primeira espécie a partir da única figura apresentada e a ilustração da segunda não corresponde à da espécie com que foi identificada. Silva (1999) descreveu e ilustrou quatro espécies (Chroomonas acuta Utermöhl, Cryptomonas erosa Ehrenberg, C. marssonii Skuja e C. platyuris Skuja) a partir de material do Lago Monte Alegre em Ribeirão Preto. Tucci (2002) estudou material do Lago das Garças, PEFI, incluindo breve descrição (com medidas) e ilustração de Chilomonas paramecium Ehrenberg, Cryptomonas brasiliensis Castro, C. Bicudo \& D. Bicudo., C. curvata Ehrenberg emend. Pennard, C. erosa Ehrenberg, C. marssonii Skuja, C. obovata Skuja, C. phaseolus Skuja, C. tenuis Pascher, C. tetrapyrenoidosa Skuja, Protocryptomonas ellipsoidea Skvortzov ex Castro et al., P. sygmoidea Skvortzov ex Castro et al., Rhodomonas lacustris Pascher \& Ruttner e Cyathomonas truncata (Fresenius) Fisch. Descrições bem mais completas (incluindo medidas) e ilustrações das mesmas espécies apresentadas em Tucci (2002) constam em Tucci et al. (2006). Por fim, Ferragut et al. (2005) forneceram medidas, ilustrações e distribuição no PEFI de três gêneros, oito espécies e uma forma de taxonômica, a saber: Chroomonas nordstedtii Hansgirg var. nordstedtii f. minor Nygaard, Cryptomonas brasiliensis Castro et al., C. erosa Ehrenberg, C. obovata Skuja, C. phaseolus Skuja, C. platyuris Skuja, C. tenuis Pascher e Pseudocryptomonas cf. subcylindrica Bicudo \& Tell.

\section{Metodologia}

A lista de espécies foi elaborada a partir de quatro fontes disponíveis, quais sejam: (1) a monografia sobre o grupo publicada pelo projeto "Flora ficológica do Estado de São Paulo (Castro \& Bicudo 2007) dentro do Programa BIOTA, (2) informações sobre o material depositado no Herbário Científico do Estado de São Paulo "Maria Eneyda P. Kauffmann Fidalgo" (SP), (3) informações disponíveis na Lista de Espécies da Flora do Brasil (Menezes \& Bicudo 2010) e (4) artigos científicos, dissertações e teses que possuem descrições e ilustrações.

\section{Resultados e Discussão}

A lista das espécies, variedades e formas taxonômicas de Cryptophyceae do Estado de São Paulo resume nove gêneros e 39 espécies, todas registradas em ambientes de água doce, incluindo sistemas lóticos, semilênticos e lênticos e tanto material fitoplanctônico quanto perifítico.

A lista é a seguinte:

Chilomonas cryptomonadoides Skuja

C. insignis (Skuja) Javornický

C. oblonga Pascher f. minor (Czosnowski) Javornický

C. paramaecium Ehrenberg

Chroomonas coerulea (Geitler) Skuja

C. nordstedtii Hansgirg f. nordstedtii

C. nordstedtii Hansgirg f. minor Nygaard

Cryptochrysis commutata Pascher

C. minor Nygaard 
C. pochmannii Huber-Pestalozzi

C. polychrysis Pascher

Cryptomonas brasiliensis Castro, C. Bicudo \& D. Bicudo

C. claviformis C. Bicudo

C. curvata Ehrenberg emend. Penard

C. cylindrica Ehrenberg

C. erosa Ehrenberg

C. lobata Koršikov

C. marssonii Skuja

C. obovata Skuja

C. ovata Ehrenberg

C. ozolini Skuja

C. phaseolus Skuja

C. parapyrenoidifera Skuja

C. platyuris Skuja

C. pyrenoidifera Geitler

C. tenuis Pascher

C. tetrapyrenoidosa Skuja

Cyanomonas americana (Davis) Oltmans

Goniomonas brasiliensis C. Bicudo

G. truncata (Fresenius) Stein var. truncata [= Cyathomonas truncata Fresenius var. truncata]

Protocryptomonas acuta Castro, C. Bicudo \& D. Bicudo

P. chilomonoides Skvortzov ex Castro, C. Bicudo \& D. Bicudo

P. ellipsoidea Skvortzov ex C. Bicudo
P. sygmoidea Castro, C. Bicudo \& D. Bicudo

Pseudocryptomonas americana $\mathrm{C}$. Bicudo \& Tell

P. parrae C. Bicudo \& Tell

P. subcylindrica C. Bicudo \& Tell

Rhodomonas lacustris Pascher \& Ruttner

R. lacustris Pascher \& Ruttner var. lacustris

Cryptomonas foi o gênero com maior número de espécies (Figura 1), também constatado por Menezes \& Novarino (2003) em seu levantamento para o Brasil. Cryptomonas erosa foi a espécie com maior número de registros (46), seguida por C. marssonii (28) e C. obovata (25) (Tabela 1).

Do levantamento realizado, cabe destacar Goniomonas brasiliensis C. Bicudo descrita recentemente por Bicudo (Castro \& Bicudo 2007) a partir de material proveniente dos municípios de Bragança Paulista (SP188324), Moji das Cruzes (SP188211) e São Carlos (SP188213). A combinação Goniomonas truncata (Fresenius) Stein var. truncata foi feita a partir de Cyathomonas truncata (Fresenius) Fisch var. truncata (SP365419), seu basiônimo, que foi citado na literatura paulista por Castro et al. (1991) e Tucci et al. (2006).

Comentários sobre a lista e riqueza do estado comparada com a de outras regiões

Dos 38 táxons identificados em Castro \& Bicudo (2007), 17 foram ali citados pioneiramente para o Brasil e, simultaneamente, para o Estado de São Paulo. São eles: Chilomonas cryptomonadoides Skuja, C. insignis (Skuja) Javornický, Chroomonas coerulea (Geitler) Skuja, C. nordstedtii Hansgirg f. nordstedtii, C. nordstedtii Hansgirg f. minor Nygaard, Cryptochrysis commutata Pascher, C. minor Nygaard, C. pochmanni Huber-Pestalozzi, C. polychrysis Pascher, Cryptomonas claviformis C. Bicudo, C. cylindrica Ehrenberg, C. lobata Koršikov, C. ozolini Skuja, C. parapyrenoidifera Skuja, C. pyrenoidifera Geitler, Cyanomonas americana (Davis) Oltmanns e Goniomonas brasiliensis C. Bicudo.

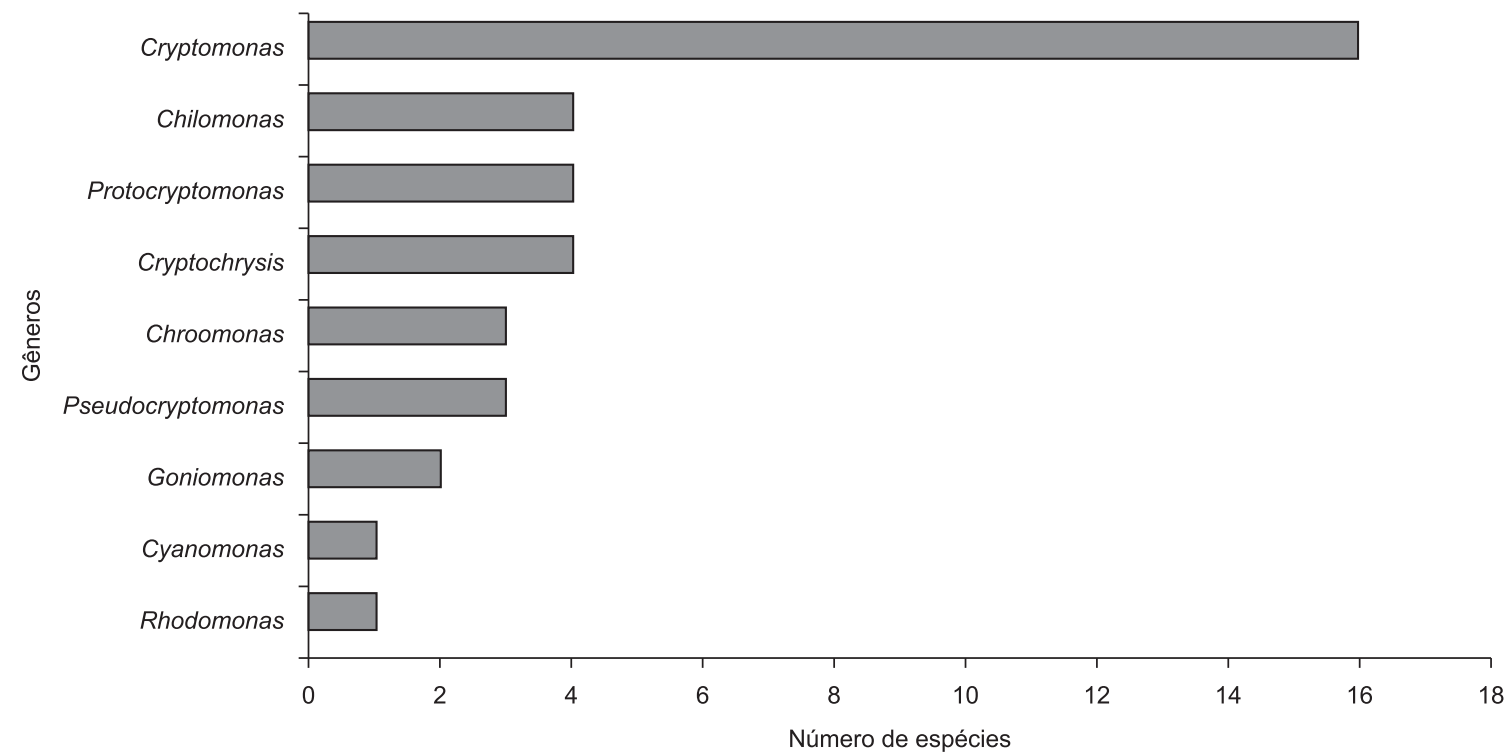

Figura 1. Número de espécies registrado em São Paulo para cada gênero de Cryptophyceae.

Figure 1. Number of species recorded in Sao Paulo for each genus of Cryptophyceae. 
Cryptophyceae do estado de São Paulo

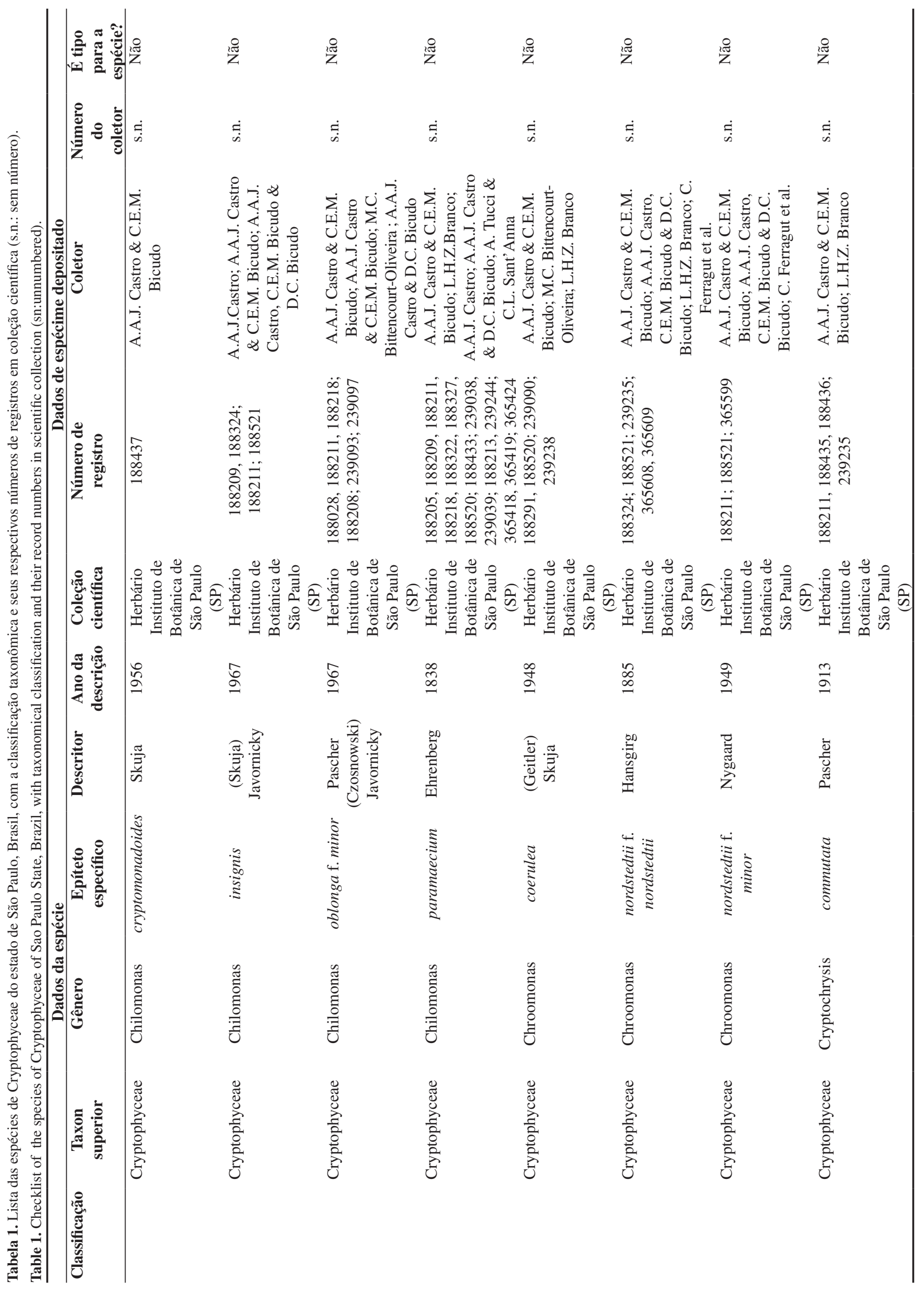


Tucci, A. et al.

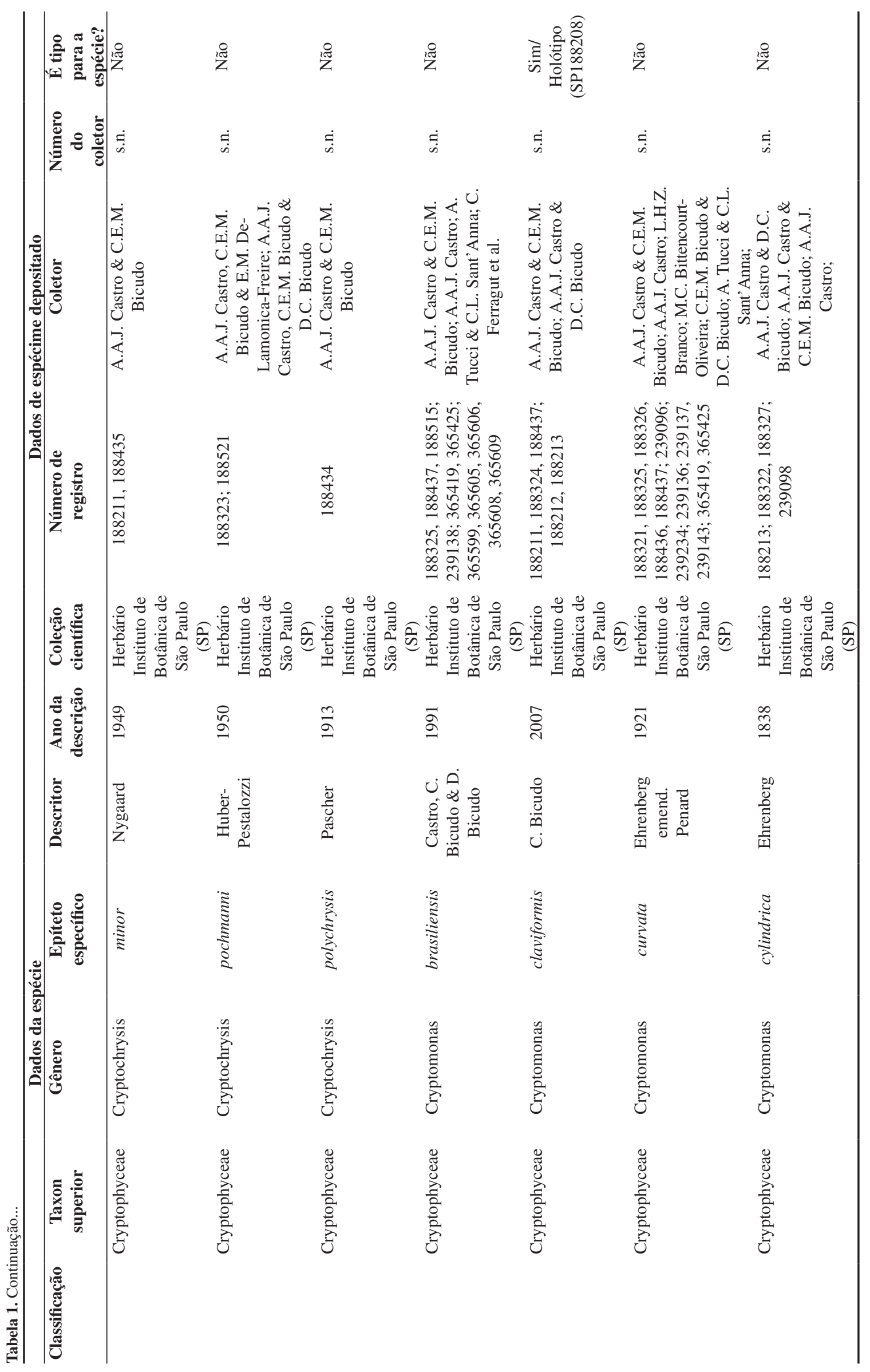




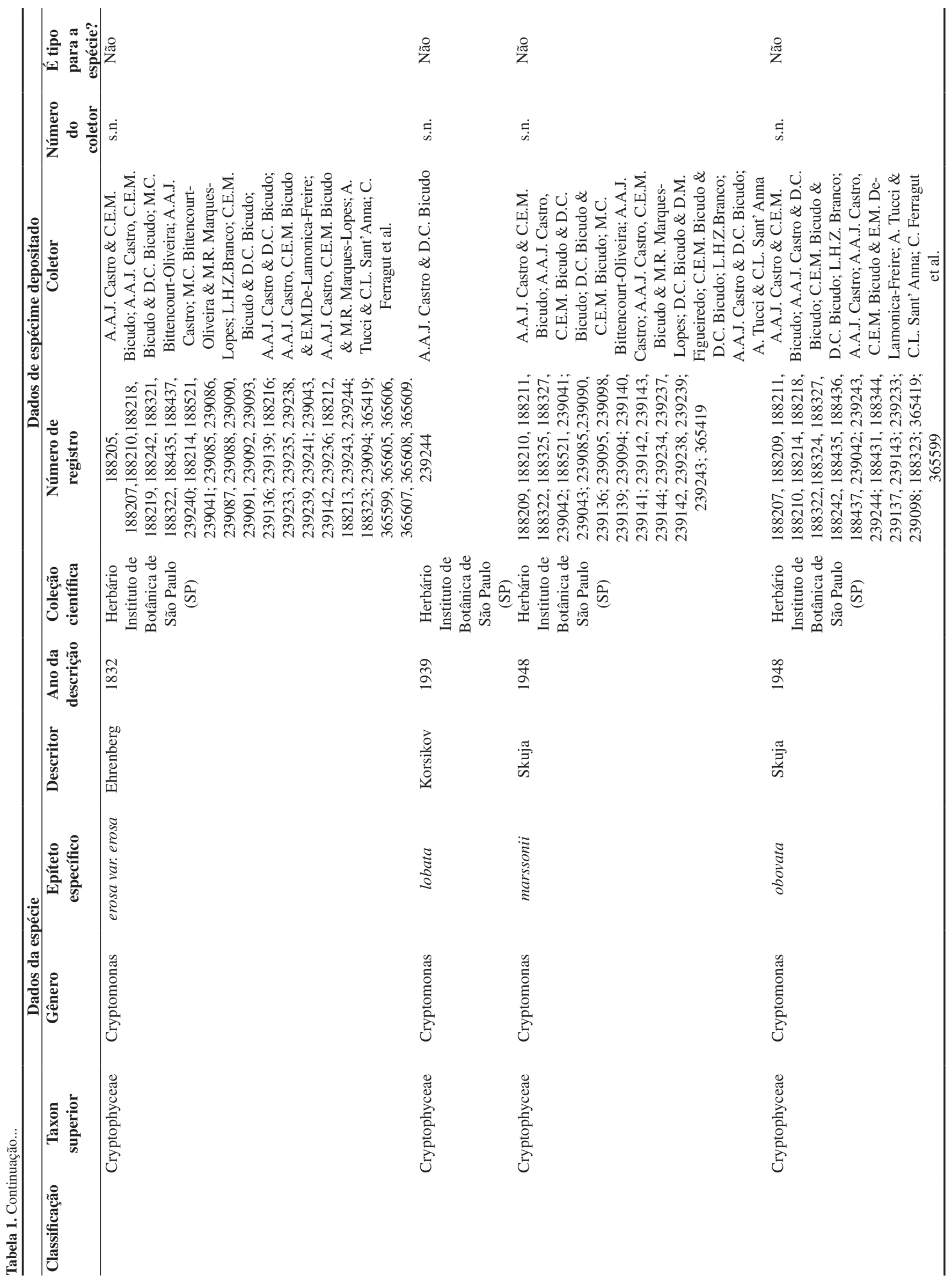


Tucci, A. et al.

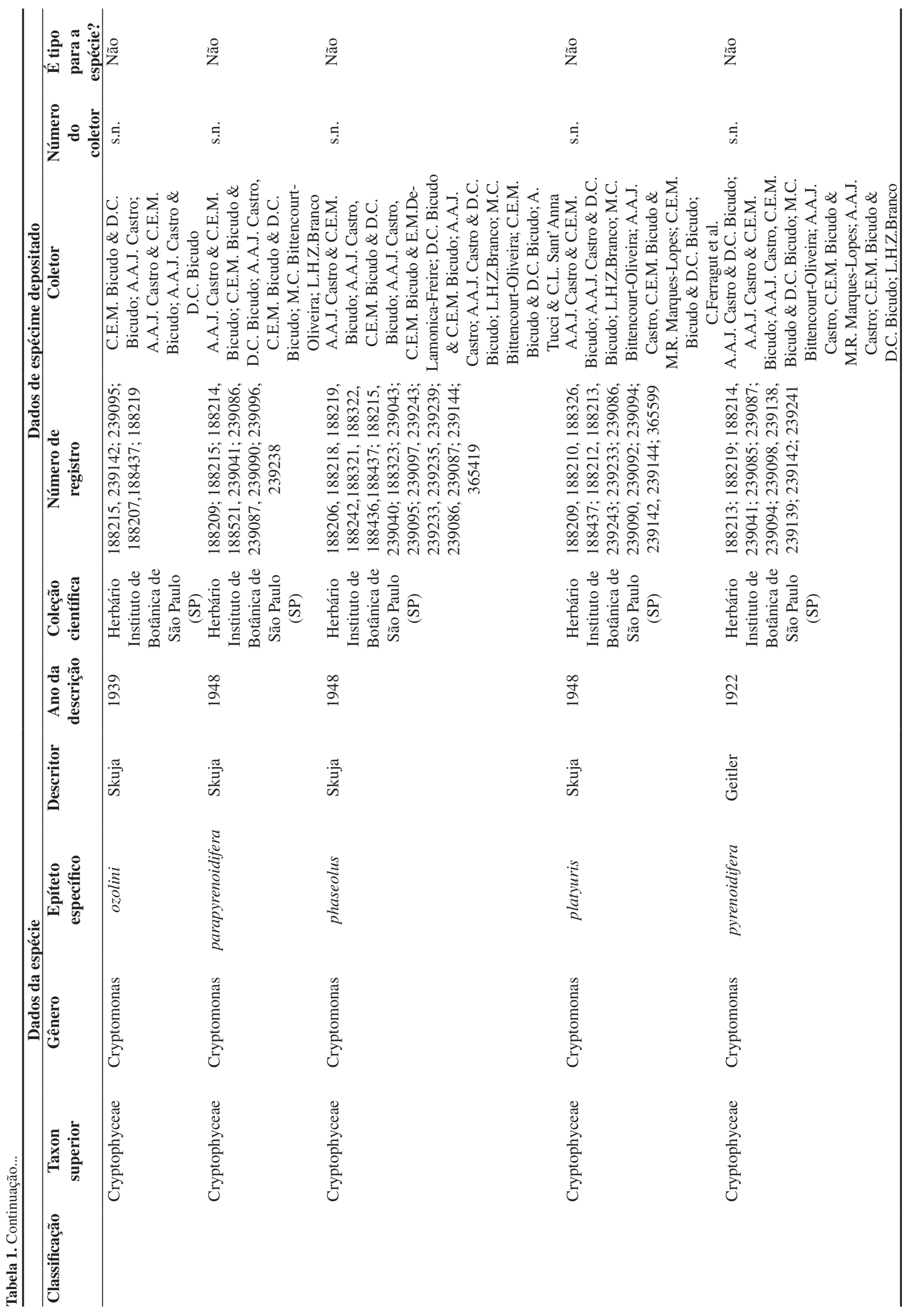




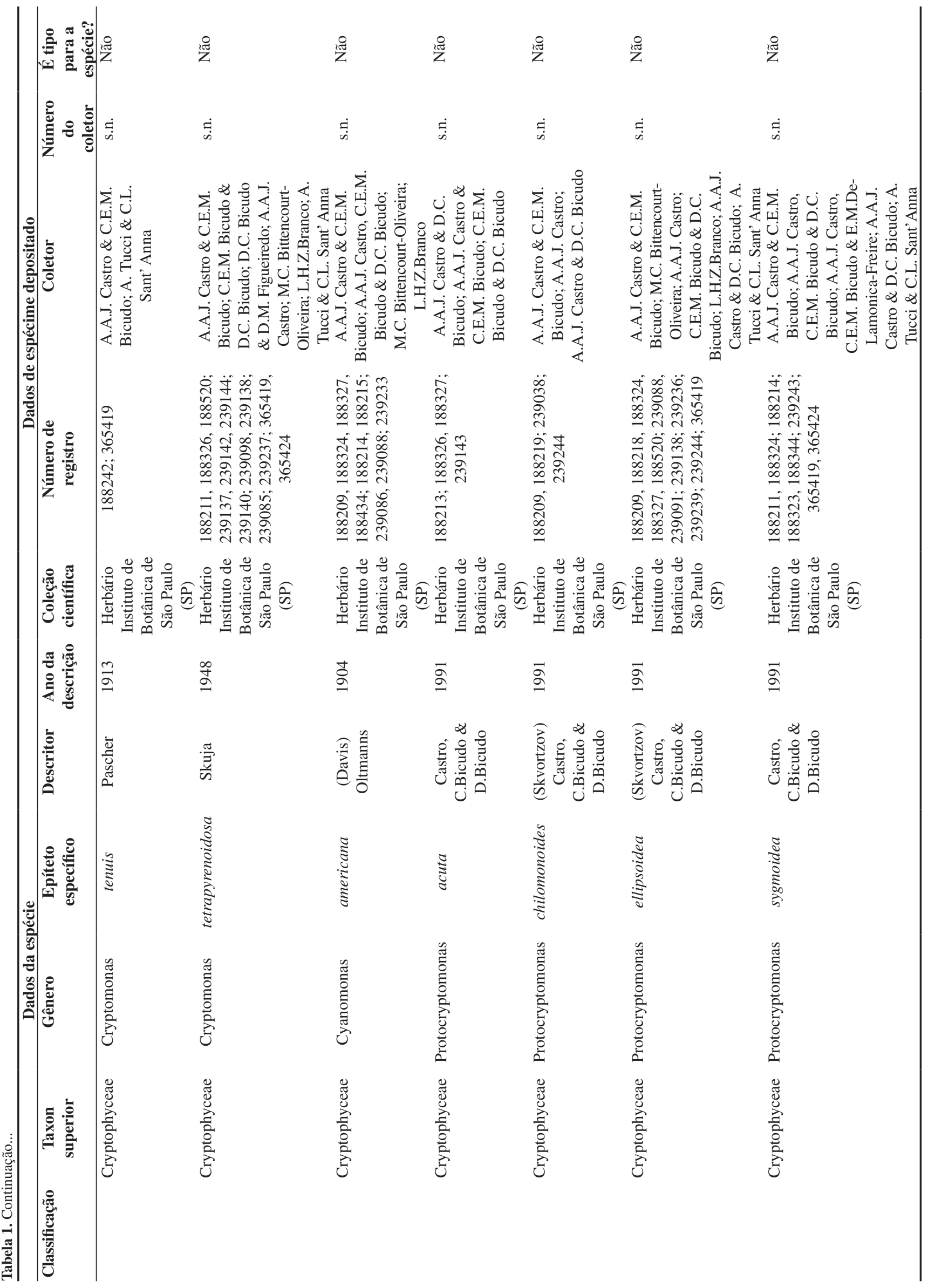


Tucci, A. et al.

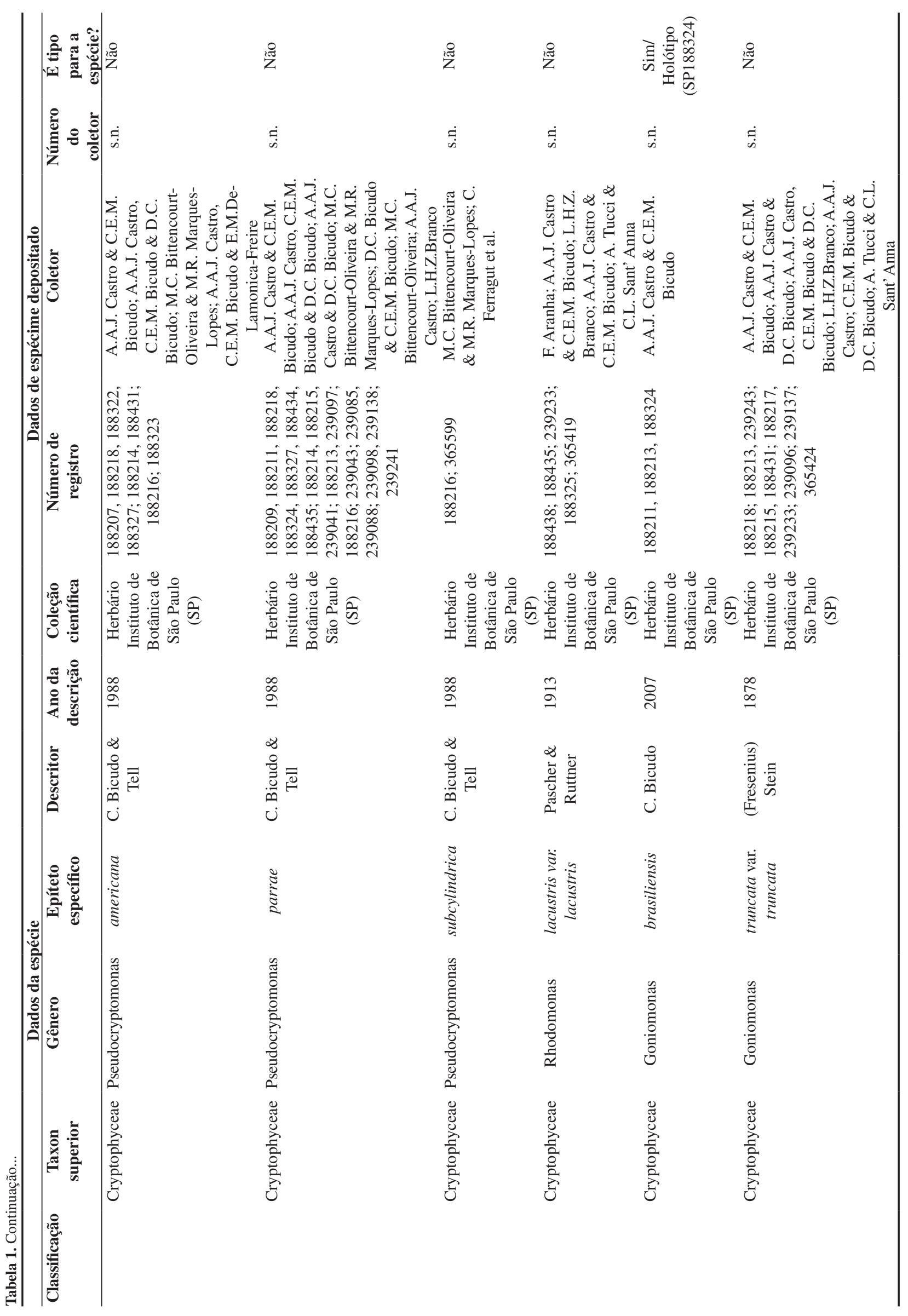


Muitos táxons descritos de material de regiões de clima temperado como, por exemplo, Chroomonas acuta Utermöhl, Chroomonas nordstedtii Hansgirg, Cryptomonas platyuris Skuja, Cryptomonas pyrenoidifera Geitler e Cryptomonas tetrapyrenoidosa Skuja foram encontradas também no Brasil. Menezes \& Novarino (2003) mencionaram que entre os 38 táxons registrados de águas tropicais brasileiras, $81 \%$ também são encontrados em regiões temperadas. Os mesmos autores também afirmaram que outras espécies tais como Cryptomonas brasiliensis descrita por Castro et al. (1991) e duas espécies de Pseudocryptomonas - P. americana e $P$. Subcylindrica - descritas por Bicudo \& Tell (1988) a partir de material coletado no Estado de São Paulo, ainda não foram encontradas fora do Brasil, sugerindo que possam ser geograficamente restritos às águas tropicais do Brasil e, possivelmente, endêmicos em alguns ecossistemas específicos.

Menezes \& Novarino (2003) comentaram que as espécies de grande porte como, por exemplo, Cryptomonas curvata Ehrenberg emend. Pennard, Cryptomonas marssonii Skuja (possivelmente aliada a Campylomonas reflexa (Skuja) Hill e Cryptomonas erosa Ehrenberg)têm sido relatados em ecossistemas aquáticos rasos eutróficos, tanto no Brasil como em regiões temperadas e, portanto, parecem ter uma distribuição cosmopolita. Esta associação pode ser comprovada pelos estudos ecológicos desenvolvidos em um dos ambientes do PEFI, o Lago das Garças, que é caracterizado por ser um sistema raso e eutrófico (Tucci et al. 2006).

Nenhum gênero de criptoficea foi registrado no litoral paulista, no levantamento realizado por Villac et al. (2008). Os autores reuniram informação de 100 anos de estudos e pesquisas sobre a composição taxonômica do fitoplâncton do litoral do Estado de São Paulo e inventariaram a biodiversidade fitoplanctônica da região. Dos 572 táxons listados, as diatomáceas foram predominantes (82\%), seguidas por dinoflagelados (16\%) e uma pequena contribuição de sílico-flagelados, cocolitoforídeos, ebriídeas e cianobactérias.

\section{Principais Grupos de Pesquisa}

São poucos os grupos de pesquisa no Brasil destinados à taxonomia de microalgas e esses se encontram distribuídos pelas regiões brasileiras sul e sudeste, com cinco e quatro grupos de pesquisas, respectivamente; regiões centro-oeste e nordeste com três grupos cada uma; e região norte com dois grupos de pesquisa. Apesar da existência desses grupos, a carência de especialistas em taxonomia de Cryptophyceae é quase absoluta. A única especialista nesse grupo de algas é a Dr ${ }^{\mathrm{a}}$ Mariângela Menezes, que trabalha no Laboratório de Ficologia, Museu Nacional, Universidade Federal do Rio de Janeiro.

A distribuição das espécies para no Brasil (Menezes \& Bicudo 2010) mostra que o Estado de São Paulo com seus três grupos de pesquisa é, sem dúvida, o que apresenta o maior número de ocorrências registradas, seguido pelo Estado do Rio de Janeiro com 13 táxons (Menezes \& Bicudo 2010). Existe então, de fato, uma lacuna muito grande nos estudos de levantamento florístico para a maioria dos estados brasileiros.

\section{Principais Acervos}

O principal acervo de material de Cryptophyceae no estado está na coleção de algas preservada em líquido (solução aquosa de formalina a 4\%) no Herbário Científico do Estado "Maria Eneyda P. Kauffman Fidalgo" (SP) do Instituto de Botânica da Secretaria do Meio Ambiente do Estado de São Paulo. São cerca de 29.000 números com representantes de algas, dos quais 339 correspondem a registros de Cryptophyceae (Figura 2), de amostras provenientes dos estados de São Paulo (95\%), Mato Grosso do Sul, Espírito Santo e Amazonas.

\section{Principais Avanços Relacionados ao Programa BIOTA/FAPESP}

O inventário florístico e o mapeamento dos táxons de criptofíceas gerados a partir dos dados levantados evidenciam que ainda há lacunas no conhecimento e demandas quanto à biodiversidade desse grupo de algas no Estado de São Paulo.

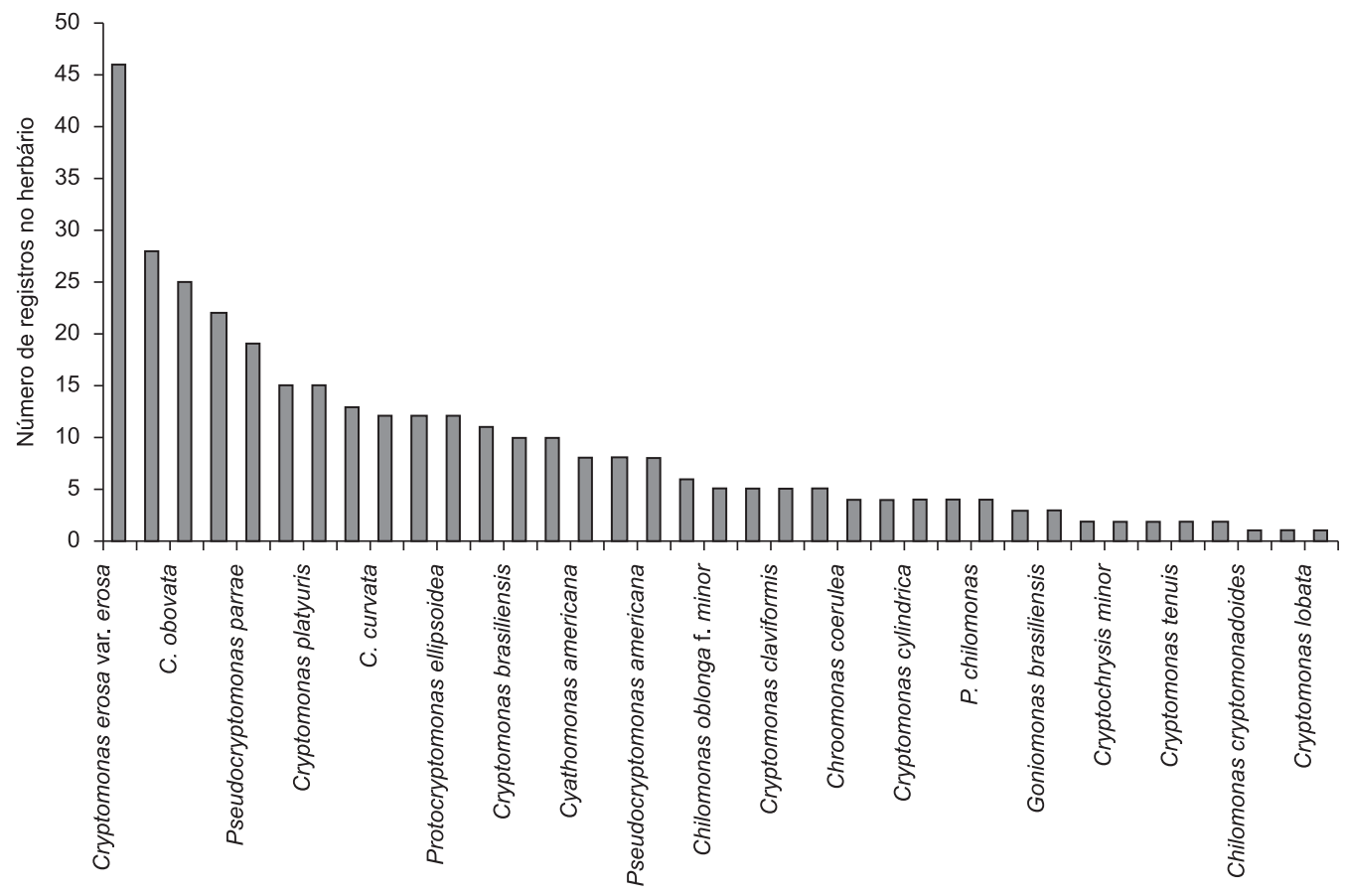

Figura 2. Número de registros no Herbário (SP) para cada espécie de Cryptophyceae registrada no estado de São Paulo.

Figure 2. Number of records in the Herbarium (SP) for each species of Cryptophyceae registered in the state of Sao Paulo. 
A grande maioria dos registros com descrições e ilustrações de espécies está restrita aos ambientes aquáticos do Parque Estadual das Fontes do Ipiranga e de localidades e municípios visitados por Castro (1993). Nada existe com relação à ocorrência de espécies marinhas.

As espécies mais bem distribuídas geograficamente foram: Cryptomonas erosa Ehrenberg, que ocorreu em 34 dos 85 municípios amostrados e C. marssonii Skuja, em 28. Os de distribuição mais restrita, isto é, que ocorrem em um único município foram os seguintes: Chilomonas cryptomonadoides Skuja, Cryptomonas polychrysis Pascher, Cryptomonas lobata Koršikov, C. tenuis Pascher e Pseudocryptomonas subcylindrica C. Bicudo \& Tell.

Dos 85 municípios examinados, 18 não apresentaram qualquer representante de Cryptophyceae, isto é, em 72 das 340 unidades amostrais escrutinadas. $\mathrm{O}$ fato de não se ter encontrado representantes da classe nesses locais pode indicar que, provavelmente, as coletas foram efetuadas em épocas não ou pouco favoráveis ao seu desenvolvimento ou que, simplesmente, o acaso não favoreceu a coleta de representantes de Cryptophyceae.

\section{Perspectivas de Pesquisa em Botânica para os Próximos 10 Anos}

A dificuldade de cobrir maior extensão continental e, principalmente, a costeira está associada à carência de especialistas no Estado de São Paulo e no Brasil, fato que seguramente prejudica a realização de inventários desse grupo de algas. Além disso, problemas de estratégia amostral e a necessidade de uso da microscopia eletrônica e, mais recentemente, da biologia molecular, comprometem o aumento de informações e alguns grupos de algas tornam-se absolutamente subestimados (Menezes \& Bicudo 2009).

Particularmente as espécies de criptofíceas são de difícil identificação devido às reduzidas dimensões e à fragilidade celular (deformam com facilidade) aspectos que, em conjunto, acarretam uma má preservação/manutenção de material em herbários, incluindo material tipo.

A segunda questão levantada por Menezes \& Novarino (2003) ainda está por resolver e é, segundo os referidos autores, uma das maiores dificuldades a impedir o desenvolvimento do estudo da diversidade de criptoficeas no Brasil. A falta de infra-estrutura adequada no país faz com que seja muito difícil para os poucos taxonomistas especialistas brasileiros a adotação de métodos de investigação que sejam eficientes e consistentes. Este fato torna, por sua vez, muito difícil a obtenção de resultados significativos no contexto de uma moderna estrutura taxonomia e sistemática baseadas em microscopia eletrônica (para mais detalhes veja Javornický (2003), Novarino (2003)).

Há, portanto, uma necessidade urgente de formação de taxonomistas, bem como o investimento em novas metodologias de estudo.

\section{Referência Bibliográfica}

BARCELOS, E.M. 2003. Avaliação do perifíton como sensor da oligotrofização experimental em reservatório eutrófico (Lago das Garças, São Paulo). Dissertação de Mestrado, Universidade Estadual Paulista, Rio Claro.

BEYRUTH, Z. 1996. Comunidade fitoplanctônica da Represa de Guarapiranga, 1991-92: aspectos ecológicos, sanitários e subsídios para reabilitação da qualidade ambiental. Tese de Doutorado, Universidade de São Paulo, São Paulo.

BICUDO, C.E.M. \& BICUDO, R.M.T. 1967. Floating communities of algae in an artificial pond in the Parque do Estado, São Paulo, Brazil. J. Phycol. 3:233-234.

BICUDO, C.E.M. \& TELL, G. 1988. Pseudocryptomonas, a new genus of Cryptophyceae from Southern Brazil. Nova Hedwigia 46:407-411.
BICUDO, C.E.M., FERRAGUT, C. \& MASSAGARDI, M.R. 2009. Cryptophyceae population dynamics in an oligo-mesotrophic reservoir (Ninféias Pond) in São Paulo, southeast Brazil. Hoehnea 36:99-111.

BIESEMEYER, K.F. 2005. Variação nictemeral da estrutura da comunidade fitoplanctônica em função da temperatura da água nas épocas de seca e chuva em reservatório urbano raso mesotrófico (Lago das Ninféias), Parque Estadual das Fontes do Ipiranga, São Paulo. Dissertação de Mestrado, Universidade de São Paulo, Ribeirão Preto.

CALIJURI, M.C. 1999. A comunidade fitoplanctônica em um reservatório tropical (Barra Bonita, SP). Tese de Docência-Livre, Universidade de São Paulo, São Carlos.

CARVALHO, M.C. 2003. Comunidade fitoplanctônica como instrumento de biomonitoramento de reservatórios no Estado de São Paulo. Tese de Doutorado, Universidade de São Paulo, Faculdade de Saúde Publica, São Paulo.

CASTRO, A.A.J. 1993. Cryptophyceae do Estado de São Paulo: inventário taxonômico. Tese de Doutorado, Universidade Estadual Paulista, Rio Claro.

CASTRO, A.A.J. \& BICUDO, C.E.M. 2007. Cryptophyceae. In Flora ficológica do Estado de São Paulo (C.E.M. Bicudo, org.). RiMa Editora, São Carlos, v.11.

CASTRO, A.A.J., BICUDO, C.E.M. \& BICUDO, D.C. 1991. Criptógamos do Parque Estadual das Fontes do Ipiranga, São Paulo, SE Algas, 2: Cryptophyceae. Hoehnea 18:87-106.

CROSSETTI, L.O. 2002. Efeitos do empobrecimento experimental de nutrientes sobre a comunidade fitoplanctônica em reservatório eutrófico raso, Lago das Garças, São Paulo. Dissertação de Mestrado, Universidade de São Paulo, Ribeirão Preto.

CROSSETTI, L.O. 2006. Estrutura e dinâmica da comunidade fitoplanctônica no período de oito anos em ambiente eutrófico raso (Lago das Garças), Parque Estadual das Fontes do Ipiranga, São Paulo. Tese de Doutorado, Universidade de São Paulo, Ribeirão Preto.

CROSSETTI, L.O. \& BICUDO, C.E.M. 2005a. Structural and functional phytoplankton responses to nutrient impoverishment in mesocosms placed in a shallow eutrophic reservoir (Garças Pond), São Paulo, Brazil. Hydrobiologia 541:71-85. http://dx.doi.org/10.1007/s10750-004-4668-7

CROSSETTI, L.O. \& BICUDO, C.E.M. 2005b. Effects of nutrient impoverishment on phytoplankton biomass: a mesocosms experimental approach in a shallow eutrophic reservoir (Garças Pond), São Paulo, southeast Brazil. Rev. Bras. Bot. 28(1):95-108. http://dx.doi.org/10.1590/ S0100-84042005000100009

FERMINO, F.S. 2006. Avaliação sazonal dos efeitos do enriquecimento por $\mathrm{N}$ e $\mathrm{P}$ sobre o perifíton em represa tropical rasa mesotrófica (Lago das Ninféias, São Paulo). Tese de Doutorado, Universidade Estadual Paulista, Rio Claro.

FERRAGUT, C. 2004. Respostas das algas perifíticas e planctônicas à manipulação de nutrientes $(\mathrm{N}$ e $\mathrm{P}$ ) em reservatório urbano (Lago do IAG, São Paulo). Tese de Doutorado, Universidade Estadual Paulista, Rio Claro.

FERRAGUT, C., LOPES, M.R.M., BICUDO, D.C., BICUDO, C.E.M. \& VERCELLINO, I. S. 2005. Ficoflórula perifítica e planctônica (exceto Bacillariophyceae) de um reservatório oligotrófico raso (Lago do IAG, São Paulo). Hoehnea 32(2):137-184.

FERREIRA, R.A.R. 2005. Estrutura da comunidade de algas perifíticas aderidas à macrófita aquática Eichhornia azurea Kunth em duas lagoas situadas na zona de desembocadura do rio Paranapanema na Represa de Jurumirim, SP. Tese de Doutorado, Universidade de São Paulo, São Carlos.

FONSECA, B.M. 2005. Diversidade fitoplanctônica como discriminador ambiental em dois reservatórios com diferentes estados tróficos no Parque Estadual das Fontes do Ipiranga, São Paulo, SP. Tese de Doutorado, Universidade de São Paulo, São Paulo.

GENTIL, R.C. 2007. Estrutura e dinâmica da comunidade fitoplanctônica de pesqueiros da Região Metropolitana de São Paulo, SP, em dois períodos do ciclo sazonal: seca e chuva. Tese de Doutorado, Instituto de Botânica, São Paulo. 
GRANADO, D.C., HENRY, R. \& TUCCI, A. 2009. Influência da variação do nível hidrométrico na comunidade fitoplanctônica do rio Paranapanema e de uma lagoa marginal na zona de desembocadura na Represa de Jurumirim (SP). Hoehnea 36:113-129.

HENRY, R., USHINOHAMA, E. \& FERREIRA, R.M.R. 2006. Fitoplâncton em três lagoas marginais ao rio Paranapanema e em sua desembocadura no Reservatório de Jurumirim (São Paulo, Brasil) durante um período prolongado de seca. Rev. Bras. Bot. 29(3):399-414. http://dx.doi. org/10.1590/S0100-84042006000300007

HOEF-EMDEN, K. \& MELKONIAN, M. 2003. Revision of the genus Cryptomonas (Cryptophyceae): a combination of molecular phylogeny and morphology provides insights into a long-hidden dimorphism. Protist 154(3-4):371-409. http://dx.doi.org/10.1078/143446103322454130

HOEF-EMDEN, K. 2007. Revision of the genus Cryptomonas (Cryptophyceae) II: Incongruences between the classical morphospecies concept and molecular phylogeny in smaller pyrenoid-less cells. Phycologia 46:402428. http://dx.doi.org/10.2216/06-83.1

JAVORNICKÝ, P. 2003. Taxonomic notes on some freshwater planktonic Cryptophyceae based on light microscopy. Hydrobiologia 502(1-2):271283. http://dx.doi.org/10.1023/B:HYDR.0000004285.50172.1f

LOPES, M.R.M. 1999. Eventos perturbatórios que afetam a biomassa, a composição e a diversidade de espécies do fitoplâncton em um lago tropical oligotrófico raso (Lago do Instituto Astronômico e Geofísico, São Paulo, SP). Tese de Doutorado, Universidade de São Paulo, São Paulo.

LOPES, M.R.M., BICUDO, C.E.M. \& FERRAGUT, C. 2005. Short term spatial and temporal variation of phytoplankton in a shallow tropical oligotrophic reservoir, southeast Brazil. Hydrobiologia 542:235-247. http://dx.doi.org/10.1007/s10750-004-8332-z

MARINHO, M.M. 1994. Dinâmica da comunidade fitoplanctônica de um pequeno reservatório raso densamente colonizado por macrófitas submersas (Açude do Jacaré, Moji Guaçu, SP Brasil). Dissertação de Mestrado, Universidade de São Paulo, São Paulo.

MENEZES, M. \& BICUDO, C.E.M. 2009. Algas: diagnóstico preliminar da biodiversidade no Brasil, BA, Brasil. In: LX Congresso Nacional de Botânica. EDUNEB, Salvador, p.59-64

MENEZES, M. \& BICUDO, C.E.M. 2010. Cryptophyceae in Lista de Espécies da Flora do Brasil. Jardim Botânico do Rio de Janeiro. http://floradobrasil. jbrj.gov.br/2010/FB114504

MENEZES, M. \& NOVARINO, G. 2003. How diverse are planktonic cryptomonads in Brazil? Advantages and difficulties of a taxonomicbiogeographical approach. Hydrobiologia 502:297-306. http://dx.doi. org/10.1023/B:HYDR.0000004287.36848.65

MOURA, A.N. 1997. Estrutura e produção primária da comunidade perifítica durante o processo de colonização em substrato artificial no Lago das Ninféias, São Paulo, SP: análise comparativa entre períodos chuvoso e seco. Tese de Doutorado, Universidade Estadual Paulista, Rio Claro.
NASCIMENTO-MOURA, A.T. 1996. Estrutura e dinâmica da comunidade fitoplanctônica numa lagoa eutrófica, São Paulo, SP. Brasil, a curtos intervalos de tempo: comparação entre épocas de chuva e seca. Dissertação de Mestrado, Universidade Estadual Paulista, Rio Claro.

NISHIMURA, P.Y. 2008. Ecologia da comunidade fitoplanctônica em dois braços da represa Billings (São Paulo-SP) com diferentes graus de trofia. Dissertação de Mestrado, Universidade de São Paulo, São Paulo.

NOGUEIRA, M.G. 1996. Composição, abundância e distribuição espaçotemporal das populações planctônicas e das variáveis flsico-químicas na Represa de Jurumirim, rio Paranapanema, SP. Tese de Doutorado, Universidade Federal de São Carlos, São Carlos.

NOVARINO, G. 2003. A companion to the identification of cryptomonad flagellates $($ Cryptophyceae $=$ Cryptomonadea). In $($ L. Naselli-Flores, J. Padisák \& M.T. Dokulil, eds.). Phytoplankton and equilibrium concept: the ecology of steady-state assemblages. Dev. Hydrobiol. 502:225-270.

PALMER, C.M. 1960. Algas e suprimento de água na área de São Paulo. Revista DAE 21(37):11-15.

RAMÍREZ, J.J.R. 1996. Variações espacial, vertical e nictemeral da estrutura da comunidade fitoplanctônica e variáveis ambientais em quatro dias de amostragem de diferentes épocas do ano no Lago das Garças, São Paulo. Tese de Doutorado, Universidade de São Paulo, São Paulo.

SANT'ANNA, C.L., SORMUS, L., TUCCI, A. \& AZEVEDO, M.T.P. 1997. Variação sazonal do fitoplâncton do Lago das Garças, São Paulo, SP, Brasil. Hoehnea 24:67-86.

SILVA, L.H.S. 1999. Fitoplâncton de um reservatório eutrófico (lago Monte Alegre), Ribeirão Preto, São Paulo, Brasil. Rev. Bras. Biol. 59:281-303. http://dx.doi.org/10.1590/S0034-71081999000200012

TUCCI, A. 2002. Sucessão da comunidade fitoplanctônica de um reservatório urbano e eutrófico, São Paulo, SP, Brasil. Tese de Doutorado, Universidade Estadual Paulista, Rio Claro.

TUCCI, A., DEBERDT, G.L.B. \& DEBERDT, A.J. 2004. Análise da comunidade de fitoplâncton do reservatório de Salto Grande (Americana, SP): uma revisão dos estudos desenvolvidos em um sistema eutrófico. In Reservatório de Salto Grande (Americana, São Paulo): caracterização, impactos e propostas de manejo (E.G Espíndola, A.M. Leite \& C.B. Dornfeld, orgs.). RiMa Editora, São Carlos, p.107-153.

TUCCI, A., SANT'ANNA, C.L., GENTIL, R.C. \& AZEVEDO, M.T.P. 2006. Fitoplâncton do Lago das Garças, São Paulo, Brasil: um reservatório urbano eutrófico. Hoehnea 33:147-175.

VERCELLINO, I.S. 2001. Sucessão da comunidade de algas perifíticas em dois reservatórios do Parque Estadual das Fontes do Ipiranga, São Paulo: influência do estado trófico e período climatológico. Tese de Doutorado, Universidade Estadual Paulista, Rio Claro.

VILLAC, M.C., CABRAL-NORONHA, V.A.P. \& PINTO, T.O. 2008. A biodiversidade do fitoplâncton do litoral do Estado de São Paulo, Brasil. Biota Neotrop. 8(3): http://www.biotaneotropica.org.br/v8n3/pt/ article+bn01908032008 